land on upper slopes and combining forestry with improved pasture for sheep on the lower hills. Added to this he stressed the need for maintaining any existing areas of native deciduous woodland, as a genetic and aesthetic rather than an immediate economic investment. Such an attitude of compromise whether on the part of the Forestry Commission or private landowners would be welcomed by all conservationists.

\section{Plasticity of the nervous system}

from Shin-Ho Chung

A Royal Society Meeting for Discussion, organised by H. B. Barlow and R. M. Gaze, was held on December 4 and 5 at the Royal Society, London.

For two days at the Royal Society, thirteen neurobiologists presented recent evidence dealing with the adaptive nature of the nervous system. Although the topics discussed ranged from the amphibian nerve-muscle preparation to the mammalian cortex, the problems posed by new discoveries converged on one central question: what are the underlying mechanisms that enable synapses in the nervous system to be changed in response to an altered external environment? This turns out to be an enormously difficult question to answer.

The simplest system in which plasticity of synaptic connections can be demonstrated is the nerve-muscle junction. It has been known for many years that muscle fibres, when deprived of their original nerves, accept newly formed branches of foreign nerves with which they do not normally connect, but the fate of such aberrant synapses when the cut nerve regenerates is under dispute. R. F. Mark (Australian National University) presented new evidence supporting his earlier claim that 'incorrect' synapses are suppressed when 'correct' synapses are reformed, rather than both remaining active. He suggests that nerve fibres compete for the available synaptic sites, and, if two nerves innervate one target cell, the less preferred one is rendered inactive. Because such a mechanism may account for several puzzling facts noted in the central nervous system, Mark's observations need to be carefully reexamined. The phenomenon of collateral sprouting, as described above, is also known to occur in the mammalian brain. G. Raisman (National Institute for Medical Research, Mill Hill) reported that vacated synaptic sites in the septal nucleus and in the superior cervical ganglion can be re-occupied by other nerves. When the vagal or hypoglossal nerve is directed to a denervated ganglion, vacated synaptic sites are occupied by the new nerve, each fibre subserving about the same number of sites as the original one.

Raisman's observations clearly demonstrate that specificity for afferent-efferent coupling in the nervous system is not rigid, and under certain conditions a foreign axon is as competent in occupying synaptic sites as the original one. If synaptic coupling between an axon and a target cell is not rigidly stipulated, it is conceivable that an axon may form a large number of aberrant contacts in the course of embryonic development. P. D. Wall (University College London) argued that even in the adult, axons have a far wider anatomical distribution than post-synaptic physiology would suggest. Wall provided evidence that an axon entering the cat spinal cord traverses and forms synaptic contacts over several spinal segments. The distant connections are normally suppressed, but their presence can be unmasked by stimulating the axon electrically. The mechanisms by which such synapses are suppressed remain unknown, as are the means by which post-synaptic cells recognise appropriate axons. There are known to be several regions in the nervous system where an axon forms a series of transient connections during development before establishing stable contacts with post-synaptic cells. M. J. Keating (National Institute for Medical Research, Mill Hill) adduced that the fibres connecting the two optic tecta in Xenopus shift their connections continually during development. Such re-adjustments are necessitated by the gradual movement of the eyes relative to the animal's head; in order to register an image seen by the two eyes onto the same area in the tectum, a series of synaptic contacts are temporarily formed and subsequently broken. He presented elegant evidence that the ability of a nerve fibre to reform new connections declines slowly with age. This discovery may be a neurophysiological basis for human learning.

A large part of the meeting was devoted to the mammalian visual system. Anatomical studies have shown that optic axons terminate in bands of cells in the geniculate body, fibres from each eye ending in alternating layers. All the axons originating from the geniculate body project onto the fourth cell layer of the visual cortex, where alternating clusters of fibres subserving one eye are interdigitated with those of the other, giving the appearance of a chequerboard pattern. From his spectacular series of anatomical studies. P. Rakic (Harvard University) elucidated the sequence of events leading to the formation of these intricate anatomical arrangements in the rhesus monkey. Cells in the different visual centres divide mitotically at different embryonic stages; those forming the geniculate body, for example, are all generated within a few days, whereas the cortical cells are continually being formed over a period of two months. Before the geniculate cells are segregated into several distinct layers, optic nerve fibres invade the region, those from one eye intermingling with those from the other. Similarly, fibres receiving the inputs from the left and right eyes do not form regularly spaced clusters but are distributed as a continuous sheet in the visual cortex. From such amorphous masses of cells and fibres, the ordered patterns of fibres carrying inputs from the right and left eyes gradually evolve.

Because visual fibres from both eyes project to the cortex, most postsynaptic visual cells respond to stimulation of either eye. When an animal is reared with one eye closed, cortical cells then respond predominantly to the eye which has been left open. C. Blakemore (University of Cambridge) showed that in the kitten only a brief period of sensory deprivation during the critical stages causes all cortical cells to be heavily dominated by the normal eye. Evidence that such an occular dominance, once patterned, is not modifiable by extensive retraining of the deprived eye later in the animal's life was provided by $\mathrm{K}$. -P. Hoffman (University of Mainz). $\mathbf{S}$. LeVay (Harvard University) demonstrated the anatomical basis of occular dominance in a rhesus monkey deprived of vision in one eye for a year. The alternating clusters of terminals from the right and left eyes in the cortex are no longer of equal size but instead clusters from the normal eye gradually expand, and those from the deprived eye shrink.

There may indeed be a common basis for all the diverse facts put forward at this meeting. If we can unravel the underlying mechanisms by which the visual centres become ordered so exquisitely, we will then understand how terminals there expand and shrink as a result of monocular visual deprivation. Similarly, if the means by which nerve cells recognise synaptic contacts as appropriate or inappropriate are elucidated, it will also become clear how nerve cells make continual adjustments and re-adjustments of their synaptic relations during development. These are problems for which neurobiologists cannot even begin to formulate questions. Answers will be difficult to find when one does not even know what questions to ask. 